\title{
Neuropathological alterations in diabetic truncal neuropathy: evaluation by skin biopsy
}

\author{
Giuseppe Lauria, Justin C McArthur, Peter E Hauer, John W Griffin, David R Cornblath
}

\begin{abstract}
Objectives-To describe the neuropathological features in skin biopsies from patients with diabetic truncal neuropathy. Methods-Three patients with diabetic truncal neuropathy underwent skin biopsies from both symptomatic and asymptomatic regions of the chest and trunk. After local anaesthesia, biopsies were performed using a $3 \mathrm{~mm}$ diameter punch device (Acupunch). Intraepidermal nerve fibres (IENFs), the most distal processes of small myelinated and unmyelinated nerve fibres, were identified after staining with PGP 9.5 as previously described.

Results-Diabetes was diagnosed at the time of the neurological presentation in two, and one was a known diabetic patient. All three had associated sensory-motor polyneuropathy. In all, skin biopsies showed a marked reduction of both epidermal and dermal nerve fibres in the symptomatic dermatomes, compared with skin from asymptomatic truncal areas. In one patient, a follow up skin biopsy when symptoms had improved showed a return of IENFs.

Conclusions-In diabetic truncal neuropathy, skin biopsies from symptomatic regions show a loss of IENFs. After clinical recovery, there is a return of the IENF population, suggesting that improvement occurs by nerve regeneration. These findings suggest that sensory nerve fibre injury in diabetic truncal neuropathy is distal to or within the sensory ganglia. Skin biopsy provides a possible tool for understanding the pathophysiology of the disease.

(F Neurol Neurosurg Psychiatry 1998;65:762-766)
\end{abstract}

Keywords: diabetic truncal neuropathy; diabetes; neuropathy; skin biopsy

Epidemiology

J C McArthur

Department of

Neuroscience, Johns

Hopkins University,

Baltimore, MD, USA

J W Griffin

Correspondence to: Dr David R Cornblath, Pathology 627, 600 North Wolfe Street, Baltimore, MD 21287-6965, USA

Telephone 001410955

2229; fax 0014105026737

Received 23 February 1998 and in revised form 19 May 1998

Accepted 1 June 1998 dorsal root ganglia or all three- and the pathogenesis.

Diabetic truncal neuropathy usually affects the middle and lower thoracic segments, either unilaterally or bilaterally, with a predilection for T8 through T12. Both abrupt and gradual onset of symptoms have been described. ${ }^{2-4} 89$ Although diabetic truncal neuropathy can occur in isolation, more often it has been reported in association with other diabetic neuropathies, particularly symmetric polyneuropathy and amyotrophy.

To our knowledge, pathological alterations in diabetic truncal neuropathy have not previously been reported. Prior studies have shown that morphological and morphometric studies of unmyelinated intraepidermal nerve fibres (IENFs) identified from a punch skin biopsy with the panaxonal marker PGP 9.5 is a useful tool for assessment of sensory fibres. ${ }^{10-13} \mathrm{We}$ report the neuropathological features in the affected skin of three patients with diabetic truncal neuropathy and compare them to findings from asymptomatic skin in the same patients.

\section{Methods}

Skin biopsies were taken from symmetric areas of the right and left back, or chest, or both, which included both symptomatic and asymptomatic regions. After obtaining informed consent, skin biopsies were performed using a 3 $\mathrm{mm}$ diameter punch device (Acupunch), after anaesthesia by local injection with $2 \%$ lidocaine with adrenalin. Tissues were processed, and IENFs identified as previously described. ${ }^{11}$ All patients underwent thorough neurological, cardiac, and gastrointestinal examinations, nerve conduction studies (NCS), needle EMG, and thoracic spine MRI.

\section{Results}

PATIENT 1

A 71 year old man developed a sharp, burning pain along the belt line, but only anteriorly. Non-insulin dependent diabetes was found, and oral hypoglycaemic medication begun. $\mathrm{He}$ had been a borderline diabetic for at least 5 years. Four months after its onset, the truncal pain suddenly disappeared. After three asymptomatic weeks, he developed mild pain over the left chest associated with a sensation of "needles and knives", worsened by touch. A heavy pressure pain then developed around the back. During this period he had hypersensitivity over the left chest from T4 to T6. Sensation to hot, cold, and pinprick were decreased in the T3-T8 area. Touching the anterior chest caused the sensation of tingling. Vibratory 
thresholds in the fingers and toes were markedly increased, with normal pinprick, touch, and position sensation. Cardiac and abdominal evaluations and thoracic spine MRI were normal. Nerve conduction studies disclosed a sensory polyneuropathy, a right median neuropathy at the wrist, and a right ulnar neuropathy at the elbow; EMG showed fibrillations and positive waves in the left T6 paraspinal muscles. Left truncal symptoms progressively improved over the next 6 months and then completely disappeared.

Skin biopsies were taken from the left and right T6 chest wall during the symptomatic phase. In the left (symptomatic) side, there was severe depletion of fibres both in the epidermis and dermis. Fibres in the dermal stratum showed an excessive segmentation. Morphological evaluation of the right asymptomatic side was normal (fig $1 \mathrm{~A}, \mathrm{~B}$ ).

Two years after diabetic truncal neuropathy onset, when symptoms had disappeared, the patient underwent a second skin biopsy from the left T6 chest wall, which disclosed an improved epidermal and dermal innervation with normal morphological features of the fibres (fig $1 \mathrm{C}$ ).

PATIENT 2

A 58 year old man developed pain over the left kidney which quickly moved up to the back and the front of the chest, from T3 to T6. The pain was described as deep, pin-like, and knife-like, and on occasion was sudden enough to make him jump. Five months after onset, diabetes was discovered, and oral antidiabetic medication begun. A similar pain then developed over the right low back, which moved superiorly and again radiated to the front, involving T3 through T8 segments. There was an area of hypoaesthesia to hot, cold, and pinprick on the left from $\mathrm{T} 3$ to $\mathrm{T} 6$ and on the right from $\mathrm{T} 3$ to T8 that extended from the midline posteriorly to the midline anteriorly, encompassing the entire dermatomes. Vibration threshold was mildly increased in the big toes. Nerve conduction studies disclosed a mild sensory polyneuropathy; EMG showed fibrillations and positive waves in the left and right T5 paraspinal muscles.

Skin biopsies were taken from the left and right back at T1 (asymptomatic) and T6-T8 segments. The T6-T8 biopsies showed a reduced number of IENFs compared with the uninvolved T1 skin biopsies. Dermal fibres appeared either thickened and dystrophic or fine and excessively segmented, such changes consistent with fibre degeneration have been seen during experimental axonal degeneration. ${ }^{14}$ At $\mathrm{T} 1$ segments, IENF density was not remarkably higher than that found at the lower thoracic dermatomes, and in the dermis only few varicose and segmented fibres were found.

PATIENT 3

A 59 year old man with non-insulin dependent diabetes for 10 years developed the abrupt onset of "burn-like"pain on the anterior chest wall at T7-T8 level bilaterally. He had severe
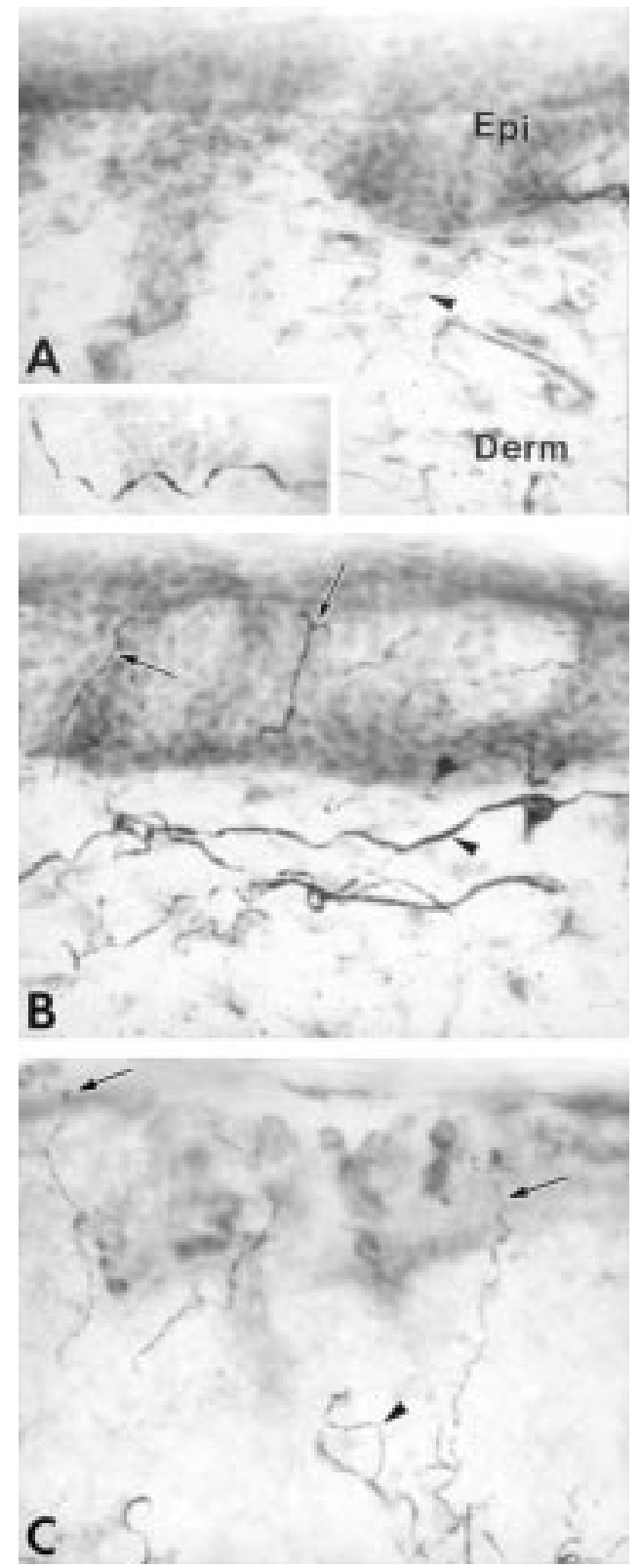

Figure 1 Photomicrographs of $50 \mu \mathrm{m}$ vertical sections of punch skin biopsy immunostained with PGP9.5 in a 71 year old patient with left T3-T8 diabetic truncal neuropathy. Epi=epidermis; Derm =dermis $(A, B$, and $C$, magnification originally $\times 310$; inset in $A$, magnification originally $\times 365$ ). (A) Left T6 chest wall biopsy showing severe reduction of epidermal and dermal nerve fibres, with excessive segmentation of residual fibres (arrowhead). In the inset, single dermal fibre from the dermis of the same symptomatic area stained with GAP43, related to its possible up regulation during regeneration processes. (B) Skin biopsy from the right asymptomatic T6 chest wall, showing epidermal (arrows) and dermal (arrow head) nerve fibres. (C) Left T6 chest wall skin biopsy 18 months after the clinical recovery, confirming the reinnervation of both epidermis (arrows) and dermis (arrowhead).

allodynia over his trunk. Acute symptoms partially recovered over the next three to four weeks, replaced by a sensation of constant itching. Then, in the same area, a sharp, needle-like pain developed, more marked on the left side. Pain showed a relapsing-remitting occurrence, with brief though severe episodes about every 30 minutes. He experienced similar pain in his right buttock, feet, and hands, along with numbness in his left thigh. Sensation was 

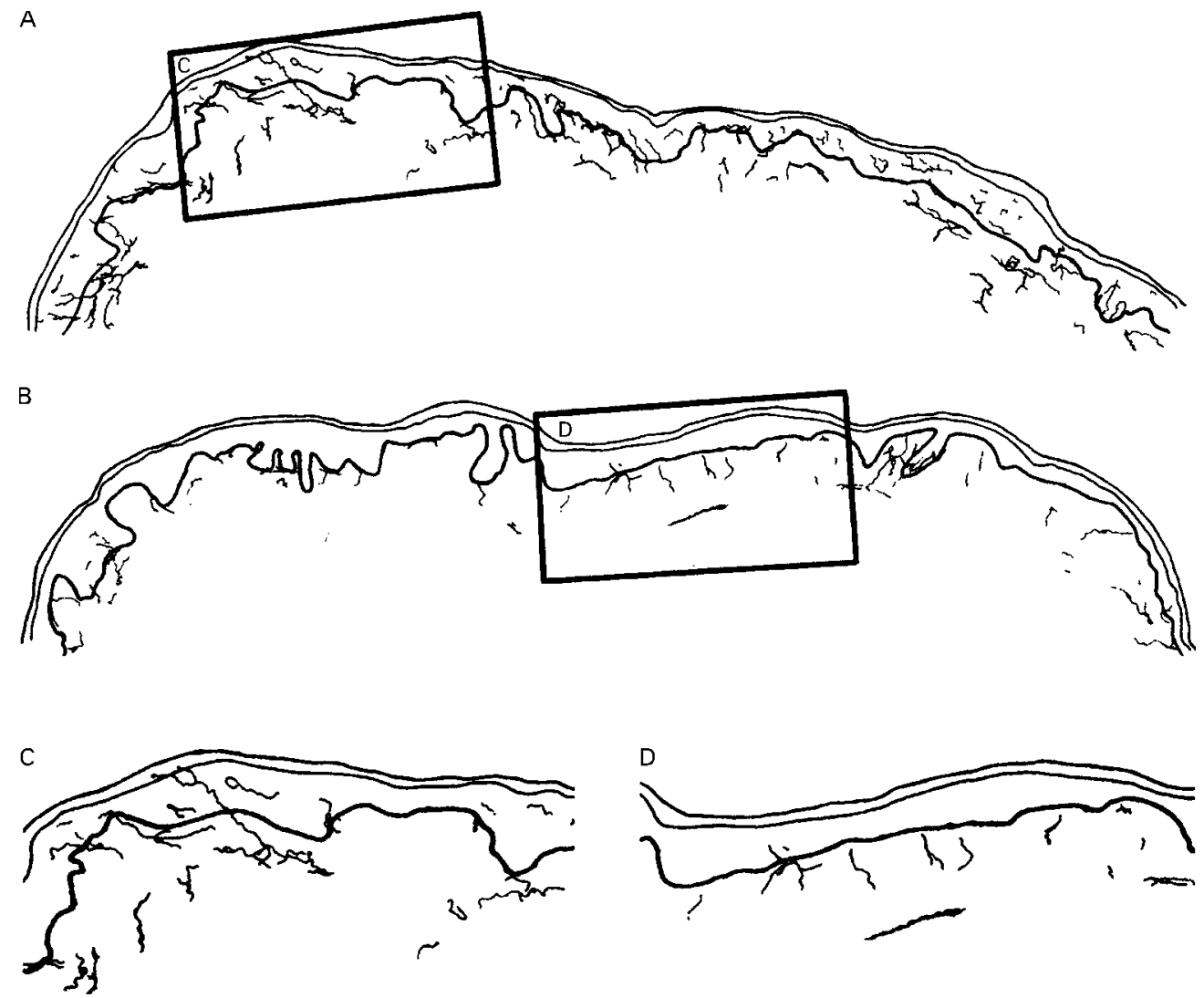

Figure 2 Drawings made by camera lucida (Olympus) of whole $50 \mu \mathrm{m}$ vertical sections of punch skin biopsy immunostained with PGP9.5 from patient 1 with left T3-T8 diabetic truncal neuropathy ( $A$ and $B$ magnification originally $x 1.25 ; C$ magnification $x 310 ; D$ magnification originallyx275). ( $A$ and $C$ (enlarged from $A$ )) Left and ( $B$ and $D$ (enlarged from $B)$ ) right T6 chest wall biopsy. Comparison between the two sides clearly shows the severe and diffuse reduction of cutaneous innervation in the symptomatic region.

decreased to touch, hot, cold, and pinprick over the trunk in the T7-8 area and over the left thigh. Vibratory threshold was increased at the toes. Nerve conduction studies disclosed a mild axonal sensory-motor polyneuropathy. Electromyography showed abnormal spontaneous activity in the right and left T7-T8 paraspinal muscles and in the L5 and, more marked, in the $\mathrm{S} 1$ roots bilaterally, although worse on the left side, consistent with a lumbosacral plexopathy. Autonomic function tests showed abnormalities in blood pressure response to grip and in heart rate variability to breathing and the Valsalva manoeuvre.

Skin biopsies were taken from the left T7 segments on back and chest wall and from the ipsilateral T4 segments. A significant reduction of both epidermal and dermal nerve fibres was seen at the left T7 dermatome, compared with the T4 skin biopsy, with an excessive segmentation of nerve fibres within the papillary dermis.

\section{Discussion}

In these three patients with diabetic truncal neuropathy, skin biopsies clearly showed that sensory fibres in areas of symptomatic skin were abnormal compared with asymptomatic skin, either contralateral homologous skin or adjacent skin. The loss of IENFs and the presence of degenerative changes of dermal nerve fibres provided an excellent correlation to the subjective sensory findings (figs 1 and 2).
Moreover, the possibility of performing a follow up skin biopsy in one of our patients also allowed us to confirm that clinical recovery correlated with the regression of the neuropathological changes (fig $1 \mathrm{C}$ ). This study demonstrates the broader use of skin biopsy as an objective measure of nerve fibre function that is minimally invasive and repeatable. ${ }^{11} 12$

Diabetic truncal neuropathy represents a distinct syndrome among diabetic patients, mostly with adult onset disease. ${ }^{146}$ In this syndrome, the predominance of sensory symptoms is typical. Symptoms that often appear abruptly, are characterised by burning or sharp pain over the back and chest, usually with a dermatomal distribution. Occasionally, abdominal wall weakness may be present. ${ }^{4515}$ In almost all the clinical series ${ }^{2-5} 9$ as well as in our patients, patients complain of frank pain, unpleasant dysaesthesias, and paraesthesias, associated with hyperpathia and allodynia. These symptoms are related to dysfunction of small myelinated and unmyelinated sensory fibres. Electromyography is useful in diagnosis to confirm the neuropathic nature of the disorder. Although the prognosis of diabetic truncal neuropathy is good, ${ }^{1}$ extensive investigations to exclude possible cardiac, gastrointestinal, and intraspinal diseases are usually performed. ${ }^{46}$ Our patients exhibited many of the features previously described in diabetic truncal neuropathy. ${ }^{134}$ In two of our cases, diabetes was diagnosed at the onset of the 
neuropathy. Cardiac and gastrointestinal evaluation were performed before the neurological diagnosis was made. Nerve conduction studies disclosed the presence of associated symmetric polyneuropathy in all patients, and autonomic function abnormalities were present in the one patient tested. Abnormalities in EMG were most prominent in symptomatic paraspinal muscles. Because of the primary impairment of small myelinated and unmyelinated fibres, nerve conduction studies alone cannot confirm the diagnosis of diabetic truncal neuropathy.

The localisation of the lesion(s) causing diabetic truncal neuropathy is still controversial. It has been suggested that the disease may develop from involvement of one or more dorsal root ganglia as well as spinal roots, mixed spinal nerves, thoracic posterior primary rami, intercostal nerves, and abdominal nerves. ${ }^{5} \mathrm{~A}$ dermatomal distribution of the symptoms would suggest a lesion of dorsal root ganglia or spinal nerves, whereas more restricted patterns would imply damage of dorsal rami or of their medial and lateral branches. Paraspinal muscle denervation suggesting involvement of motor fibres would localise the lesion to the nerve root, spinal nerve, ventral ramus, or anterior horn cell level. In addition, lesions involving different sensory and motor fibres within the spinal nerve roots could be postulated. ${ }^{5}$

Recent experimental findings ${ }^{16}$ disclosed that IENF density significantly decreased after either dorsal root ganglionectomy or sciatic nerve transection in rats, but did not after dorsal and ventral radiculotomy, sympathectomy or spinal motor neuron lesion. Thus the finding in all our patients of abnormal epidermal innervation within the symptomatic regions suggests a primary lesion of either dorsal root ganglia, spinal nerve, or peripheral nerve; the presence of paraspinal denervation suggests that the lesion is either in spinal roots or peripheral nerves before the branches to paraspinal muscles exit. Without a necropsy evaluation of a symptomatic patient, the precise localisation of the lesion(s) will remain controversial.

The pathogenesis of the syndrome remains unknown. It has been suggested that patients with rapid and limited onset have a multifocal vascular disorder, whereas those with gradual and more widespread symptoms could be affected by a metabolic dying back process. ${ }^{16}$ The possible mechanism(s) causing pain in diabetic neuropathies is still debated. In particular, the relation between symptoms and nerve morphology has been controversial. Impulses arising from damaged or regenerating small myelinated and unmyelinated axons have been suggested to be the generators of pain. ${ }^{17}{ }^{18}$ However, the number of degenerating or regenerating axons documented by nerve biopsy did not correlate with painful symptoms in several studies. ${ }^{19-22}$ Investigations of experimental peripheral nerve injury have shown that after $\mathrm{A} \delta$ and $\mathrm{C}$ fibres had broken down, these primary afferents became spontaneously active and had lowered thresholds to physiological stimuli. ${ }^{23}$ Similar evidence has been reported in human peripheral nerve injury. ${ }^{24}$ Transient injury of distal nerve fibres can induce a temporary CNS sensitisation which, once established, maintains the secondary hyperalgesia with only a low level of C nociceptor inputs. ${ }^{26}{ }^{27}$ We have recently shown that in patients with idiopathic painful small fibre neuropathies and distal small fibre sensory loss, there is loss of the most distal terminals of small myelinated and unmyelinated fibres. ${ }^{28}$ Lastly, Rowbotham and Fields ${ }^{29}$ found a strong positive correlation of allodynia severity, pain, and preservation of thermal sensation in postherpetic neuralgia patients, suggesting that functioning small diameter afferent nociceptors are required to generate pain. Further studies of the morphological and physiological correlates of pain are clearly needed.

This work was supported by The Donald and Rosalie Dietz and The James Dunn Funds for Neuromuscular Research, NS 26643, RR 00722, and the Blaustein Pain Treatment Program

1 Thomas PK, Tomlinson DR. Diabetic and hypoglycemic neuropathy. In: Dyck PJ, et al, eds. Peripheral neuropathy. Vol 2. Philadelphia: WB Saunders, 1993:1219-50.

2 Ellenberg $M$. Diabetic truncal mononeuropathy-a new clinical syndrome. Diabetes Care 1978;1:10-13.

3 Sun SF, Streib EW. Diabetic thoracoabdominal neuropathy: clinical and electrodiagnostic features. Ann Neurol 1981;9: $75-9$.

4 Kikta DG, Breuer AC, Wilbourn AJ. Thoracic root pain in diabetes: the spectrum of clinical and electromyographic findings. Ann Neurol 1982;11:80-5.

5 Stewart JD. Diabetic truncal neuropathy: topography of the Stewart JD. Diabetic truncal neuropathy: to
sensory deficit. Ann Neurol 1989;25:233-8.

6 Wilbourn AJ. Diabetic neuropathies. In: Brown WF, et al, eds. Clinical electromyography. London: Butterwortheds. Clinical electromyography.

7 Shulz A. Diabetische Radiculapathie der Unteren Tarakalsegmente mit Bauchdeckenparesen. Verh Dtsch Ges Inn Med 1966;72:1171-5.

8 Longstretch GF, Newcomber AD. Abdominal pain caused by diabetic radiculopathy. Ann Intern Med 1977;86:16686. $9 \begin{aligned} & \text { Massey EW. Diabetic truncal mononeuropathy: electromyo- } \\ & \text { graphic evaluation. Acta Diabetologica Latina 1980;17:269- }\end{aligned}$ 72.

10 Levy DM, Terenghi G, Gu X-H, et al. Immunohistochemical measurements of nerves and neuropeptides in diabetic skin: relationship to tests of neurological function. Diabetologia 1992;35:889-97.

11 McCarthy BG, Hsieh S-T, Stocks EA, et al. Cutaneous innervation in sensory neuropathies: evaluation by skin biopsy. Neurology 1995;45:1848-55.

12 Holland NR, Stocks EA, Hauer P, et al. Intraepidermal nerve fiber density in patients with painful sensory neuropathy. Neurology 1997;48:708-11

13 Kennedy WR, Wendelschafer-Crabb G, Johnson T. Quantification of epidermal nerves in diabetic neuropathy. Neurology 1996;47:1042-8.

14 Hsieh S-T, Choi S, Lin W-M, et al. Epidermal denervation and its effects on keratinocytes and Langerhans cells. $\mathcal{f}$ Neurocytol 1996;25:513-24.

15 Boulton AJM, Angus E, Ayyar DR, et al. Diabetic thoracic polyradiculopathy presenting as abdominal swelling. BMf 1984;289:798-9.

16 Li Y, Hsieh S-T, Chien H-F, et al. Sensory and motor denervation influence epidermal thickness in rat foot glabrous skin. Exp Neurol 1997;147:452-62.

17 Asbury AK, Fields HL. Pain due to peripheral nerve damage: an hypothesis. Neurology 1984;34:1587-90.

18 Brown MJ, Martin JR, Asbury AK. Painful diabetic neuropathy: a morphometric study. Arch Neurol 1976;33: neuropa $164-71$.

19 Llewelyn JG, Gilbey SG, Thomas PK, et al. Sural nerve morphometry in diabetic autonomic and painful sensory neuropathy: a clinicopathological study. Brain 1991;114: 867-92.

20 Dyck PJ, Lambert EH, O'Brien PC. Pain in peripheral neuropathy related to rate and kind of fiber degeneration. Neurology 1976;26:466-77.

21 Britland ST, Young RI, Sharma AK, et al. Association of painful and painless diabetic polyneuropathy with different patterns of nerve fiber degeneration and regeneration. Diabetes 1990;39:898-908.

22 Britland ST, Young RJ, Sharma AK, et al. Acute and remitting painful diabetic polyneuropathy: a comparison of ting painful diabetic polyneuropathy: a comparison

23 Tanelian DL, MacIver MB. Analgesic concentrations of lidocaine suppress tonic A-delta and $\mathrm{C}$ fiber discharges produced by acute injury. Anesthesiology 1991;74:934-6. 
24 Cline MA, Ochoa J, Torebjork HE. Chronic hyperalgesia and skin warming caused by sensitized C nociceptors.

25 Koltzenburg M, Torejbork HE, Wahren LK. Nociceptor modulated central sensitization causes mechanical hyperalgesia in acute chemogenic and chronic neuropathic pain Brain 1994;117:579-91

26 Simone DA, Baumann TK, LaMotte RH. Dose-dependent pain and mechanical hyperalgesia in humans after intradermal injection of capsaicin. Pain 1989;38:99-107.
27 Simone DA, Sorkin LS, Oh U. Neurogenic hyperalgesia: central neural correlates in responses of spinothalamic tract neurons. F Neurophysiol 1991;66:228-46.

28 Holland NR, Crawford TO, Hauer P, et al. Small-fiber sensory neuropathies: Clinical course and neuropathology of idiopathic cases. Ann Neurol 1998; (in press).

29 Rowbotham MC, Fields HL. The relationship of pain, allodynia and thermal sensation in post-herpetic neuralgia. Brain 1996;119:347-54

\section{NEUROLOGICAL STAMP}

\section{Hermann von Helmholtz (1821-94)}

In the fune 1998 issue of the fournal, this vignette was inadvertently published with the wrong stamp. It is reproduced here with the correct stamp.

The physician Hermann von Helmholtz, born in Potsdam, was, on his mother's side, a descendent of William Penn, founder of Pennsylvania. His contributions to science-which included physiology, optics, electrodynamics, and meteorology - were numerous.

His doctoral thesis, begun in 1842, was on the connection between nerve fibres and nerve cells and he graduated from the Medical School in 1843. His demonstration in isolated preparations that muscles are the main source of animal heat, led him to his best known discovery, the law of conservation of energy and his paper in 1847 Über die Erhaltung der Kraft (on the conservation of force). In 1850 he became the first to measure the velocity of nerve impulses in the sciatic nerves of frogs using a pendulum myograph of his own invention. He invented the ophthalmoscope (1851) and was the first person to see the living human retina. This was followed by his phakoscope and ophthalmometer (1852). With the latter he was able to explain the mechanism of accommodation (1854), particularly the part played by the lens. His research on the eye and the result of his extensive research was his multivolume Handbuch der physiologischen Optik (Handbook of physiological optics) published in 1867, was one of the great contributions to medicine in the 19th century. The handbook was regarded as a classic and described by Von Graefe as "the Bible of the ophthalmologist. It remained for many decades the definitive study of the physiology and physics of vision. With Thomas Young (1773-1829) of London he developed a theory of colour vision and in studies of the ear Helmholtz showed how the cochlea resonates for different frequencies and analyses complex sounds into harmonic components. In 1863 he published Die Lehre von den Tönenpfindungen als Physiologische Grundlage für die Theorie der Musik (The sensation of tone as a physiological basis for the theory of music). In this work he demonstrated that the aesthetics of music was a function of the ears' mechanical ability to pick up wave motions of musical sounds. It became the handbook of not only audiologists, physiologists, and physicists, but musicians as well. Steinway, already famous for the quality of his grand pianos, tried out the improvements suggested by Helmholtz on the Helmholtz family grand piano.

With his close friend Sir William Thompson, afterwards Lord Kelvin, he estimated the age of the sun and calculated the energy radiated from its surface. Earlier, about 1840

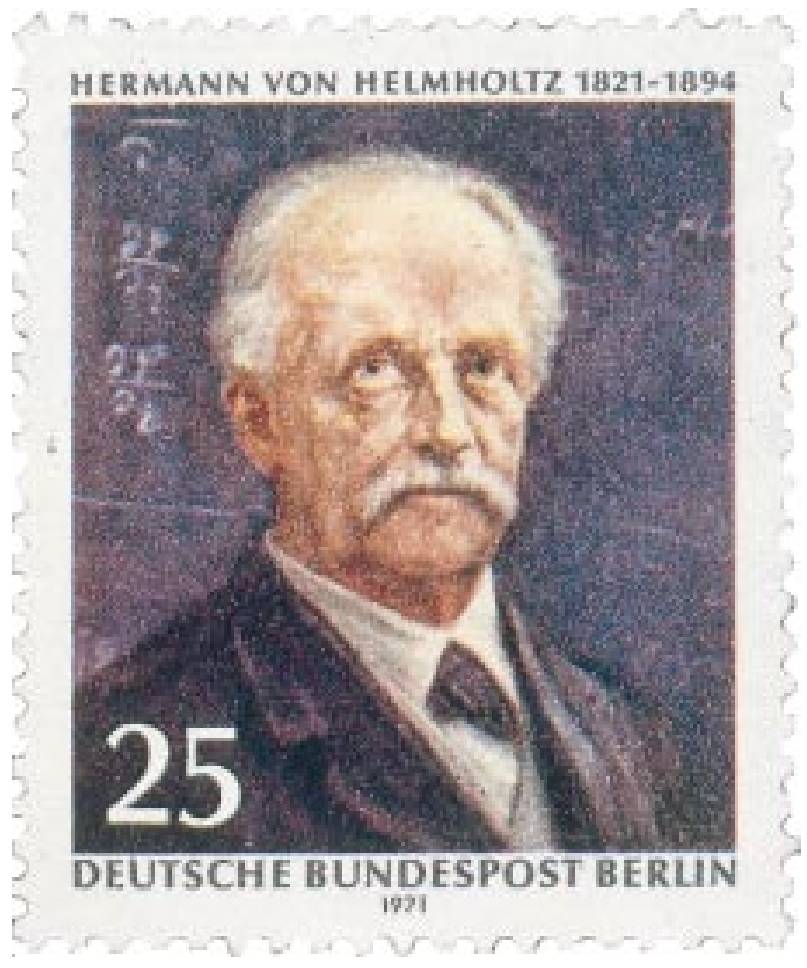

with James Prescott Joule, he had demonstrated that electric circuits obey the laws of conservation of energy and that electricity is a form of energy.

He had been at various times Professor of Physiology at Königsberg University (appointed in 1849), Professor of Anatomy (1856-66) primarily at the University of Heidelberg, and in 1871 he was appointed Professor of Physics in Berlin where he spent the rest of his life. He then turned his attention to electrodynamics, and was assisted by Heinrich Hertz, whose discovery of "Hertzian waves" made modern wireless transmission possible. Had he done nothing else in his life but invent the ophthalmoscope, his name would not be forgotten. He became one of the greatest of scientists, who did not forget that he was a physician. "Medicine", he said "was once the intellectual home in which I grew up: and even the emigrant best understands and is best understood by his native land."

He was honoured philatelically by Germany (West Berlin) in 1971 on the 150th anniversary of his birth (Stanley Gibbons B394, Scott GN314).

L F HAAS 